\title{
Exploring Google's Platform Services with Augmented Reality Apps in Cultural Tourism Context
}

\author{
Ana Dhëmbi \\ Department of Informatics \\ Faculty of Natural Sciences \\ University of Tirana \\ Tirana, Albania
}

\begin{abstract}
Over the past decade, mobile applications have revolutionized every sector of the economy, including cultural tourism. In this aspect, research in the cultural tourism field has reaffirmed this sector as a major element in the global economy. Also, research trends and recent development of technology has shifted and reshaped cultural tourism by connecting user activity to technological devices and by defining a new perceptual experience. The modern tourist experience is tied to digital geographic awareness, site accessibility and is expanded in numerous directions by the impact of new technologies.
\end{abstract}

Augmented Reality applications present users with an interactive method to collect information from real-world environments where surroundings are enhanced by computergenerated content, based on GPS information, sensors, and distance to real objects. Today Google Cloud Platform is seen as an efficient way to use cloud computing services that run on the same infrastructure. Spatial data, maps, and Augmented Reality are some of the tools available.

This paper will discuss how Google's Platform services can enhance user Augmented Reality experience in mobile applications. In consideration of Augmented Reality capabilities and GIS services, a demonstration of how Google Software Development Kit (SDK) known as ARCore functions without any additional hardware, is composed, and merges its features in mobile applications. Bringing ARCore together with AR Android Applications is part of a work-inprogress that not only helps tourists scan various venues around their surroundings but also city-wide small businesses and other tourism subsectors.

\section{Keywords}

Mobile Applications, Augmented Reality, Google's Platform, Cultural Tourism, Android Applications, ARCore, Software Development Kit.

\section{INTRODUCTION}

Cultural tourism is one of the oldest forms of international travel aimed at experiencing local heritage, landscapes, art, lifestyle, and tradition. Because culture is undoubtedly connected to tourism, visiting historical sites, seeking traditional cuisines, and meeting new people provide an important motivation for travel. [1] According to OECD (2009), cultural tourism is one of the largest and fastestgrowing global tourism markets. In 2007, around $40 \%$ of international tourism was occupied by cultural tourism. [2]

Over the past decade's technology has experienced tremendous growth and development. Technology and technological infrastructure have produced solutions and products that improve and ease people's life. The tourism system is inevitably influenced by the diffusion of information and communication technologies (ICTs). [3] The process of integration between cultural tourism and ICT has developed a new modern and hybrid concept that helps tourists have an enjoyable experience and ease the planning process. The development of this technology has altered the perceptual experience of tourism.

People are exposed to a wide range of technologies, indeed, mobile technologies, monitor-based interfaces, systems that overlay virtual objects on top of the real world are some of today's innovations. Among endless possibilities to provide software products for cultural tourism, it's a venture based on mobile devices for their portability, interactivity, programmability, and the sense of the "one stop shop". With more than two billion active devices, Android is the largest mobile platform in the world. [4] The focus is on a specific group of mobile devices, those who use Android operating systems.

Mobile devices are equipped with integrated listeners, usually, referred to as sensors. Intelligent devices (smartphones) use sensors to continuously collect different types of user information, such as visual or location-based information. Augmentation techniques are applied in real-time and in semantic context with environmental elements, by overlaying supplemental information. [5] And it hasn't been long since Google has launched its framework, ARCore, for augmented reality applications on Android phones, as an open-source service.

Based on past work [6], the purpose of this paper is to explore how an open-source AR tool can be used in AR Android applications to enhance cultural tourism.

This paper is organized as follows: In Section 1 presents AR concepts, components and techniques. Section 2 presents a general theoretical background to Google's Platform and ARCore. Section 3, presents some aspects of the integration of ARCore to an AR Android application. The final section (Section 4) concludes the main findings of this paper.

\section{AUGMENTED REALITY CONCEPTS AND COMPONENTS}

Physical reality is enriched with digital information and visualized through a computer or mobile screens. Creating AR applications over time is made easier. But creating a welldesigned AR application needs to have a better understanding of concepts, modules, and techniques used.

\subsection{AR Concepts}

AR applications use displays to overlay and merge the 
physical information with digital information, also known as visual augmentation. There are three techniques for showing visuals in AR. They are categorized as Headset AR or Handheld AR. Headsets are classified as optical see-through (OST) and video see-through (VST). Some examples of Headset devices include Google Glasses or Hololens.

Handheld AR is a more preferred method because it uses smartphones or tablets to show AR content. They are also referred to as sensor-based AR. They use a location and orientation sensor from the mobile device. Location sensor is supported by GPS, which is present in most mobile devices. Orientation sensors are identified as accelerometers, magnetometers, and gyroscopes.

Spatial see-through is the next step to a $3 \mathrm{D}$ free space technique using plasma in the air and retinal displays which projects directly on users' retina. [7] Figure 1, presents the relation between discussed techniques and the position of the medium over the real-world.

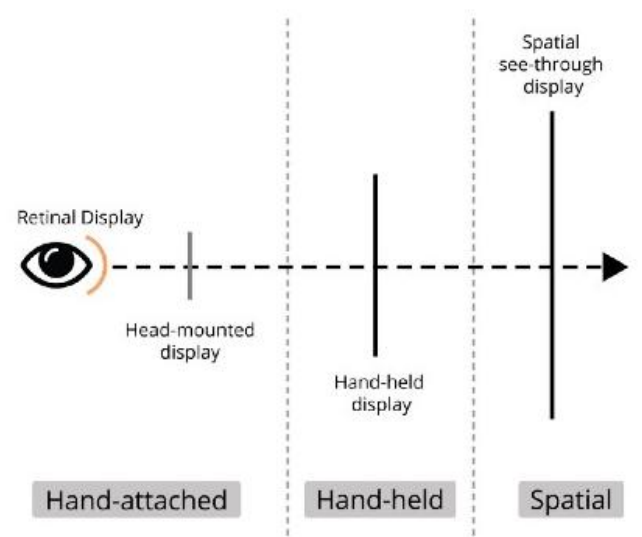

Fig 1: Augmented content comparison. [8]

AR applications use the device camera to access real-world environments, and scene generators which can be software suitable to render virtual information.

The last one is the tracking system, which is one of the most important issues on AR systems mainly due to registration problems [8]. Objects of the real and virtual world must be properly aligned with each other or the new augmented reality will be compromised.

Handheld AR uses both location and orientation sensors to track information and register virtual objects in physical scenes.

\subsection{AR Application Components}

A layered model (Fig. 2) can be a suitable definition to describe AR application elements. They are organized in three main levels: the application layer, the AR layer and operating system layer. The first layer, is built with application logic and features. AR layer is composed of all the concepts discussed above. The OS layer components are camera, sensors, screen and graphics. Sometimes they are identified as third parties and their role is not to create AR functionalities but to enable the AR layer.

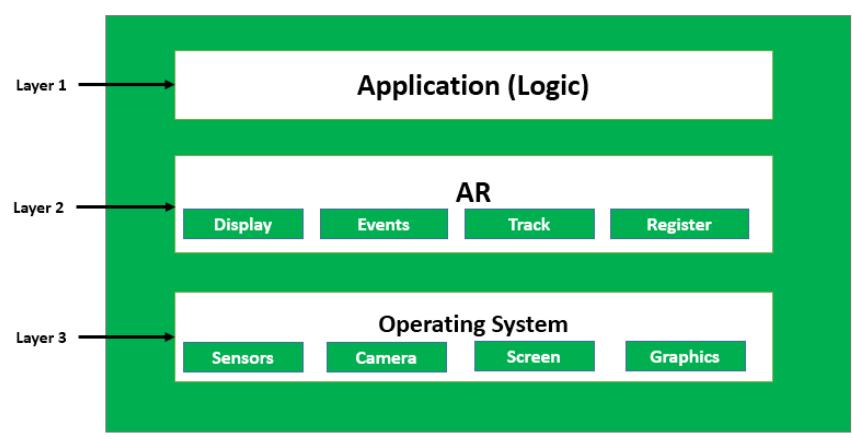

Fig 2: AR components using three layer model

\subsection{AR Techniques}

AR results are produced based on some input captured in realtime by the camera, user distance to other objects, and user orientation. AR tools use different techniques to produce results based on ways they manage AR module elements such as calculating distance or tracking, registering spatial objects, etc. There are three types of techniques used in AR tools: I) Computer vision-based, II) Sensor-based, III) Hybrid tracking.

The first technique uses the power of a built-in camera to continuously capture real-world objects, process the image, and applies computer vision algorithms to analyze it and identify objects. The information generated this way is related to the object's position. Direct object recognition may be difficult and unstable, but the deployment of markers improves the performance of the recognition system. [11] Overall this process is associated with performance issues and drains the battery of the device.

Sensor-based tracking techniques use algorithms that are based on user location, provided by device GPS, camera orientation, and movement, to track point of interest (POIs) and estimate distances between them. This technique is more accurate in outdoor environments.

In the third group, are categorized those AR services that combine both techniques, vision-based and sensor-based to track objects.

\section{GOOGLE CLOUD PLATFORM}

Over the last years, the IT industry has shifted focus to business needs and how technology can provide solutions to manage, store, compute data, network, or even host applications. They approached a new methodology by separating system components, and creating independent elements that can be used alone or easily integrated with other services.

The decision to use a physical or a dedicated host has challenged many businesses. But when it comes to some of the most powerful names in the technology sector, like Google, Amazon, Microsoft, etc., choosing cloud was undoubtedly better in many aspects, like performance, agility, lower costs, wide geographical coverage, and scalability. [12]

Providers of cloud services rely on technically different Application Programming Interfaces (APIs). Understanding which API model is the right one to incorporate into the cloud isn't always easy. Integration to an infrastructure is exposed to different methodologies and each one has its own components. The main areas of a Cloud API are as follows:

a. PaaS (Platform-as-a-Service) APIs - These are the 
service-level APIs, used to facilitate accessibility to a specified cloud environment or use cloud functionalities. A PaaS cloud API model is considered a good choice when it comes to building a platform on cloud resources.

b. SaaS (Software-as-a-Service) APIs - These APIs are called 'on-demand-software' because cloud providers manage the infrastructure and the platform that runs the application. [12] Generally, this model is integrated in ERP solutions or CRM applications.

c. IaaS (Infrastructure-as-a-Service) APIs - They control infrastructure, cloud resources and how they are distributed in low-level details. IaaS focus is in network configurations, virtual machines management, load balancers etc.

In Figure 3 is a layered stack, from Wikipedia [12], to the three main models discussed above.

Cloud computing is necessary to make use of the cloud development tools and environment to enhance AR apps functionalities.

Google Cloud Platform (GCP) was launched in 2011, but has become a unique services supplier over the years by providing massive-scale services including machine learning and artificial intelligence.

Today, GCP is one of the most important cloud services in the market, even rivaling the market leader Amazon Web Services (AWS). It enables several products for developers to build a range of programs from simple websites to complex world-wide distributed applications. [13]

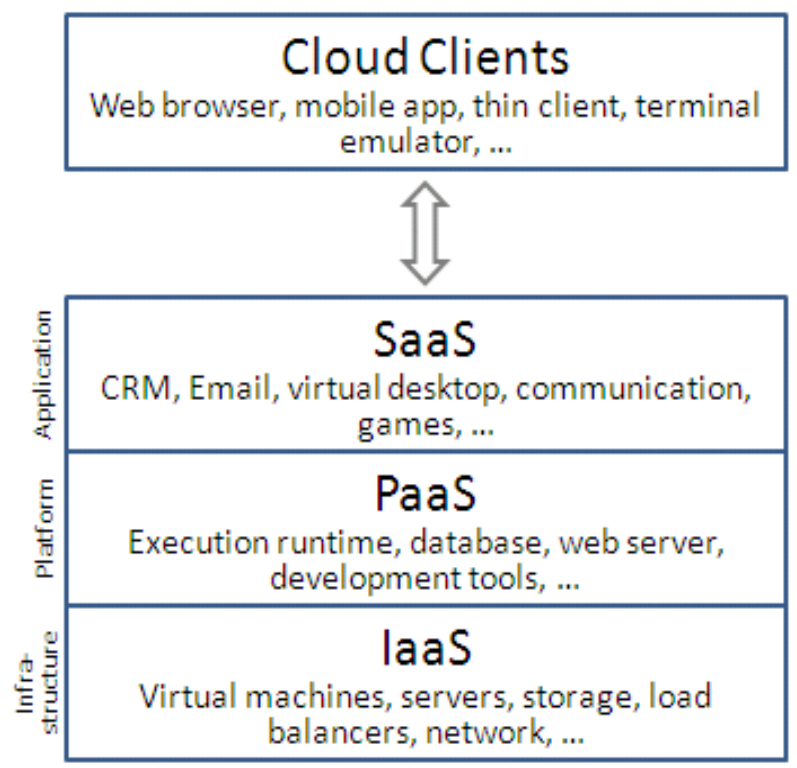

Fig 3: Cloud APIs models as layered stack.
Google Cloud products are grouped into four categories: compute, storage, big data and services. Compute is composed of Compute Engine serves as IaaS and App Engine is Google's SaaS. Storage contains three sub-categories, Cloud SQL is a relational MySQL database, used to manage and store data. Cloud Storage is an object storage service, and Cloud Datastore is a database schemaless to store nonrelational data. Big-Query is part of the Big Data category, used to analyze big data in the cloud. Services are cloud endpoints, that through RESTful services can communicate with Android, iOS, or JavaScript clients.

Google provides the so-called API Client Libraries for all services and different programming languages like Java, .NET, Python, Ruby, PHP, Node.js, Dart. GCP APIs run as subdomains of googleapis.com, for example Google Maps API subdomain is maps.googleapis.com. GCP APIs provide JSON, HTTP or gRPC interfaces through a virtual private network. Clients send HTTP and gRPC requests directly to Cloud API endpoints. [14] Cloud APIs can be accessed in many ways, through server applications, mobile apps or thirdparties. Also, they are easily accessed via Google Cloud SDK or UI Console. API Library is accessible via UI Console. All the available APIs can be discovered via the API Library interface.

ARCore is Google's platform for building Augmented Reality experiences. Using different APIs, it enables the device to do the following: sense its environment, understand the world, and interact with information. [15] There are three main capabilities of ARCore to interact virtual world to the real one through the mobile camera: tracking, environmental understanding, and light estimation. In the next section, we will discuss in technical terms how ARCore is used to provide the AR experience in Android AR Apps. 


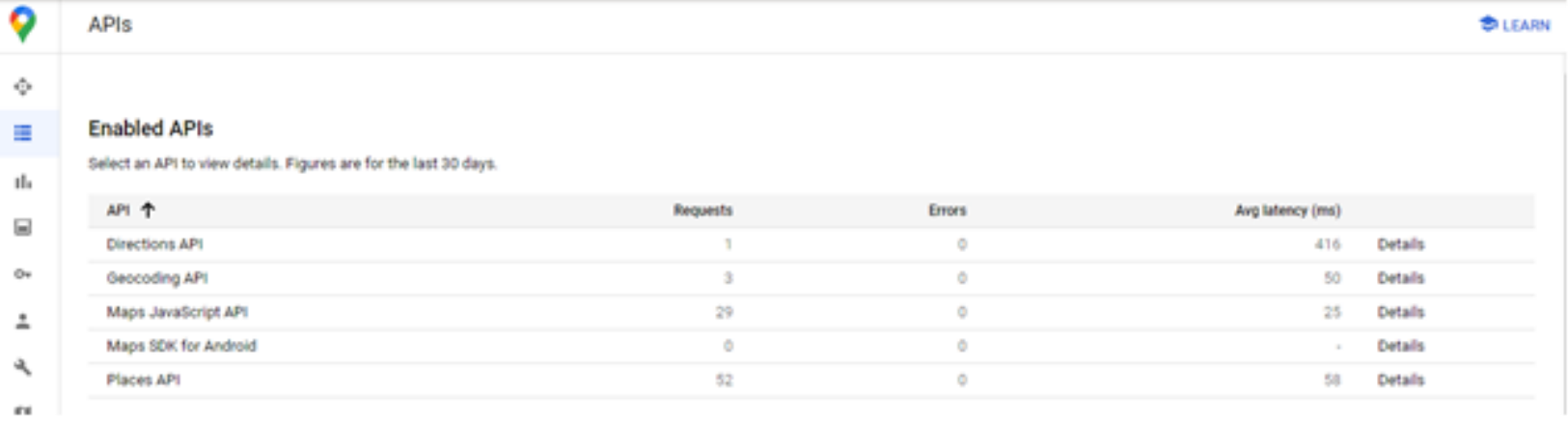

Fig 5: Enabling GCP APIs

$$
\begin{aligned}
& \text { Name * } \\
& \text { API key } 1
\end{aligned}
$$

Fig 6: GCP APIs Key

\section{AR IN ANDROID APPS USING ARCORE}

As explained in Section 1 and 2, this research uses Handheld $\mathrm{AR}$ devices and the location-based techniques to display tourists points of interests based on their current geographic position and camera orientation. In this context, one has to start with the configuration of the GCP Dashboard. GCP APIs is used to retrieve and provide geographic information for nearby places and from GPS, and then ARCore functionalities on Android apps to register this data on the camera view.

Utilizing GCP console user interface is an easy way to create the new billing project, further enabling the services needed from maps. It will be necessary to enable Google Maps SDK for Android API and Places API. See the list of the enabled GCP APIs in Figure 5.

The API key is a unique identifier that authenticates requests associated with your project for usage and billing purposes. At least one API key is required to be associated with the project. [16] Once the API key is generated (Figure 6), one can retrieve the JSON dataset of places nearby a specific location. Information about Points of Interests (PoIs) is the response of an HTTP Request where API key and location components (latitude and longitude) are some of the parameters that need to be specified.

The requested URL, to retrieve the PoIs near the center of Tirana, Albania, would be:

https://maps.googleapis.com/maps/api/place/nearbysearch/jso n?location $=41.3275,19.8187 \&$ radius $=500 \&$ types $=$ point_of_in terest\&key=AIzaSyB0P1B_IRah27-G3gwgpGJhRCdHNGrrN4

The response is formatted in JSON and below is described a part of the data about "Tirana International Hotel \& Conference Center" as an object near the city center.

$\{\ldots$

"icon":

"https://maps.gstatic.com/mapfiles/place_api/icons/v1/png_71

/lodging-71.png",

"name" : "Tirana International Hotel \& Conference Center",

"place_id" : "ChIJ0YeQ4g8xUBMRtkN9jTaRd3g",

"plus_code" : \{

"compound_code" : "8RH9+WC Tirana, Albania", "global_code" : "8FHX8RH9+WC"

\} ,

"rating" : 4.3,

"reference" : "ChIJ0YeQ4g8xUBMRtkN9jTaRd3g",

"scope" : "GOOGLE",

"types" : [

"night_club",

"parking",

"spa",

"lodging",

"bar",

"restaurant",

"food",

"point_of_interest",

"establishment"

],

"user_ratings_total" : 918,

"vicinity" : "Sheshi Skënderbej 8, Tirana"

$\ldots\}$

Once the dataset is prepared and configured work begins on the Android application and how ARCore is used to interact with the data. Displaying PoIs on the camera scene is a process that goes on the following operations [15]:

a. AR Setup - PlacesARFragment is used to manage camera permission from the user, but also to display the AR scene. PlacesARFragmet a subclass of ARFragment contained in MainActivity. Once the application is running is possible to look around the environment and detect a group of white dots over the horizontal plan, which are proof of a successful setup of AR on the Android application.

b. Getting Geographical Information - Connection with Google Maps Platform API key to set up the map, and get the user location and position it on the map requires the implementation of the FusedLocationProviderClient class method called getCurrentLocation. Nearby PoIs are made available by implementing nearbyPlaces method with parameters such as, location, radius and places type.

c. Bring data on the AR scene - Whenever the plane is detected on the AR scene an event listener is fired to 
create new anchor's, or anchor nodes which get connected to the AR scene created in the beginning.

d. Positioning Places - In order to understand the device's position in space position sensors (geomagnetic and accelerometer) are used. Event listeners are registered on sensors and managed by a SensorManager.

Implementation of the above steps on an Android application display markers on the screen while the environment is scanned.

\section{CONCLUSION}

Work presented in this paper is inspired by a work-in-progress that studies the AR technologies and their integration in mobile applications in order to improve the tourist experience. Using open-source tools and services to build AR applications is one of the main benefits of trusting Google Cloud Platform despite the fact of producing a solution without engaging additional technologies. GCP configuration and enabling tools such as Places API provided geographical data collection based on current user location. In addition our study experiments a new way to perceive AR experience using ARCore as part of GCP tools by stepping the process into the main tasks to place geographical data into user camera. Future focus of this study is to enrich application features using a variety of GCP services that helps the tourism sector, to compare and understand the difference between them.

\section{REFERENCES}

[1] G.Richards, "Cultural Tourism A review of recent research and trends", Journal of Hospitality and Tourism Management, (12-21), 2018

[2] OECD (2009) The Impact of Culture on Tourism. OECD, Paris
[3] O. Hadžić ,TOURISM AND DIGITIZATION OF CULTURAL HERITAGE (2004)

[4] D.Bruke (2017), Google AR and VR: Augmented reality at Android scale

[5] Introducing Virtual Environments National Center for Supercomputing Applications, University of Illinois.

[6] A.Dhembi, E. Xhina, D.Kavroudakis, C.Kalloniatis: Enchancing Cultural Tourism Experiences in Albania through Mobile Augmented Reality Applications, 3rd Euro-Mediterranean Conference, 2018

[7] N.Yadav, "Understanding display techniques in Augmented Reality", Medium, 2018

[8] R. Azuma. Tracking requirements for augmented reality. Communications of the ACM, 36(7):50-51.

[9] R. Silva, J. C. Oliveira, G. A. Giraldi, "Introduction to Augmented Reality"

[10] J.Grubert , R.Grasset, Book "Augmented Reality for Android Application Development"

[11] A. Bernardos, J.Cano, J.Iglesias and J.Casar, "MOBILE INDOOR AUGMENTED REALITY”, 2011

[12] Wikipedia- Cloud Computing. en.wikipedia.org/wiki/Cloud_computing

[13] S.Challita, F.Zalila, Ch.Gourdin, P.Merle. A Precise Model for GoogleCloud Platform. IEEE International Conference on Cloud Engineering - IC2E 2018, Apr 2018, Orlando, Florida, United States

[14] Google Cloud - cloud.google.com

[15] ARCore - developers.google.com

[16] Google Maps Platform developers.google.com/places/web-service 